\title{
New Nuclear Equation of State for Core-Collapse Supernovae with the Variational Method
}

\author{
H. Togashi ${ }^{1, a}$, S. Yamamuro ${ }^{2}$, K. Nakazato ${ }^{2}$, M. Takano ${ }^{1,3}$, H. Suzuki ${ }^{2}$, and K. Sumiyoshi ${ }^{4}$ \\ ${ }^{1}$ Department of Pure and Applied Physics, Waseda University, 3-4-1 Okubo Shinjuku-ku, Tokyo 169-8555, \\ Japan \\ ${ }^{2}$ Department of Physics, Faculty of Science and Technology, Tokyo University of Science, Yamazaki 2641, \\ Noda, Chiba 278-8510, Japan \\ ${ }^{3}$ Research Institute for Science and Engineering, Waseda University, 3-4-1 Okubo Shinjuku-ku, Tokyo 169- \\ 8555, Japan \\ ${ }^{4}$ Numazu College of Technology, Ooka 3600, Numazu, Shizuoka 410-8501, Japan
}

\begin{abstract}
We report the current status of our project to construct a new nuclear equation of state (EOS) with the variational method for core-collapse supernova (SN) simulations. Starting from the realistic nuclear Hamiltonian, the EOS for uniform nuclear matter is constructed with the cluster variational method: For non-uniform nuclear matter, the EOS is calculated with the Thomas-Fermi method. The obtained thermodynamic quantities of uniform matter are in good agreement with those with more sophisticated Fermi Hypernetted Chain variational calculations, and phase diagrams constructed so far are close to those of the Shen-EOS. The structure of neutron stars calculated with this EOS at zero temperature is consistent with recent observational data, and the maximum mass of the neutron star is slightly larger than that with the Shen-EOS. Using the present EOS of uniform nuclear matter, we also perform the 1D simulation of the core-collapse supernovae by a simplified prescription of adiabatic hydrodynamics. The stellar core with the present EOS is more compact than that with the Shen-EOS, and correspondingly, the explosion energy in this simulation with the present EOS is larger than that with the Shen-EOS.
\end{abstract}

\section{Introduction}

The realistic equation of state (EOS) for hot dense nuclear matter is essential in the studies of highenergy astrophysical phenomena, such as core-collapse supernovae ( $\mathrm{SNe}$ ), cooling of neutron stars (NSs) and black hole formations. However, it is a hard task to construct a SN-EOS, since a SN-EOS must cover an extremely wide range of densities $\rho$, proton fractions $Y_{\mathrm{p}}$ and temperatures $T$. In fact, only a limited number of SN-EOSs are currently available, e. g., the Lattimer-Swesty EOS [1] and the Shen-EOS [2]. Since these SN-EOSs are based on phenomenological models for uniform matter, it is desirable that the EOSs calculated with microscopic many-body theories are applied to SNe. Under these circumstances, we are now constructing a new nuclear EOS for SN simulations using the variational many-body theory starting from the realistic nuclear forces. In this project, we construct the EOS of uniform matter with the realistic nuclear Hamiltonian using the cluster variational method

a e-mail: hajime_togashi@ruri.waseda.jp 
$[3,4]$, and the EOS of non-uniform matter with the Thomas-Fermi (TF) calculation following the method by Shen et al [2]. In this paper, we report the current status of our project together with applications of the uniform nuclear EOS to NSs and SNe.

\section{Construction of the nuclear EOS for supernovae}

For uniform nuclear matter, we start from the nuclear Hamiltonian composed of the AV18 two-body potential and UIX three-body potential. At zero temperature, we calculate the expectation value of the two-body Hamiltonian with the Jastrow wave function $\Psi$ in the two-body cluster approximation to obtain the two-body energy per nucleon $E_{2}$. Here, $\Psi$ consists of the two-body correlation function $f_{i j}$ and the degenerate Fermi-gas wave function $\Phi_{\mathrm{F}}$ at zero temperature. $f_{i j}$ includes the spin-isospindependent central, tensor and spin-orbit components $\left(f_{\mathrm{C}}, f_{\mathrm{T}}, f_{\mathrm{SO}}\right)$, and $\Phi_{\mathrm{F}}$ is specified by the occupation probabilities of single-nucleon states $n_{0 i}(k)(i=\mathrm{p}, \mathrm{n})$. Then, $E_{2}$ is minimized with respect to $f_{\mathrm{C}}, f_{\mathrm{T}}$ and $f_{\mathrm{SO}}$ with appropriate constraints so that the obtained $E_{2}$ reproduces the results for symmetric nuclear matter and neutron matter with the more sophisticated Fermi Hypernetted Chain (FHNC) variational method by Akmal et al. [5] (APR). The three-body energy per nucleon $E_{3}$ based on the UIX is calculated somewhat phenomenologically so that the total energy per nucleon $E=E_{2}+E_{3}$ reproduces the empirical saturation point [4].

At finite temperatures, we calculate the free energy per nucleon $F$ of uniform nuclear matter using an extension of the variational method proposed by Schmidt and Pandharipande [6]. In this method, $F$ is expressed as the sum of the approximate internal energy per nucleon $E_{\mathrm{T}}$, which is composed of the two-body part $E_{2 \mathrm{~T}}$ and the three-body part $E_{3 \mathrm{~T}}$, and the approximate entropy per nucleon $S$. Here, $E_{2 \mathrm{~T}}$ is obtained by replaceing $n_{0 i}(k)$ in $E_{2}$ at zero temperature by the average occupation probabilities at finite temperature $n_{i}(k)$, which are parameterized by the effective masses $m_{i}^{*}(i=\mathrm{p}, \mathrm{n})$ for protons and neutrons. For simplicity, $E_{\mathrm{T} 3}$ is chosen to be the same as $E_{3} . S$ is also expressed with $n_{i}(k)$ as in the case of the non-interacting quasi-nucleon gas where the mass of quasi-nucleon is $m_{i}^{*}$, and then $F$ is minimized with respect to $m_{i}^{*}$. The obtained $F$ for symmetric nuclear matter and neutron matter are in good agreement with those with the FHNC calculation [7]. It is also found that the internal energies per nucleon derived from $F$ through the thermodynamic relation for various $\rho, Y_{\mathrm{p}}$ and $T$ are in excellent agreement with $E_{\mathrm{T}}$, which implies that the present variational method is self-consistent.

For non-uniform nuclear matter, we adopt the Thomas-Fermi (TF) method used by Shen et al [2]. In this method, we assume that a spherical nucleus is located at the center of a Wigner-Seitz (WS) cell in the BCC lattice. As $\rho$ increases and/or $Y_{\mathrm{p}}$ decreases, neutrons drip out of the nucleus at zero temperature: At finite temperatures, the drip of protons also occurs. Furthermore, appearance of $\alpha$ particles outside the nucleus is taken into account. Here, we assume that the $\alpha$ particles are classical particles and each has a fixed volume. Then the free energy of a WS cell is expressed as the sum of the nuclear part and the contribution from the $\alpha$ particles. The nuclear part consists of the bulk term, gradient term and Coulomb term. In the bulk term, the free energy density of the uniform hot asymmetric nuclear matter calculated with the variational method explained above is adopted. The gradient term represents a part of the surface energy, and its strength is determined so that the similar TF calculations for isolated atomic nuclei reproduce the gross feature of their masses and radii [8]. The last term represents the Coulomb energy caused by the non-uniform charge distributions in the WS cell. These three terms are expressed with the distribution of $\mathrm{p}, \mathrm{n}$ and $\alpha$. Their density distribution functions in the WS cell are parameterized, and then the total free energy density of a WS cell is minimized with respect to those parameters.

The TF calculations for non-uniform matter is now in progress: At typical temperatures, the phase diagrams (the non-uniform region and the $\alpha$-mixed region) obtained so far are similar to those of the 
INPC 2013

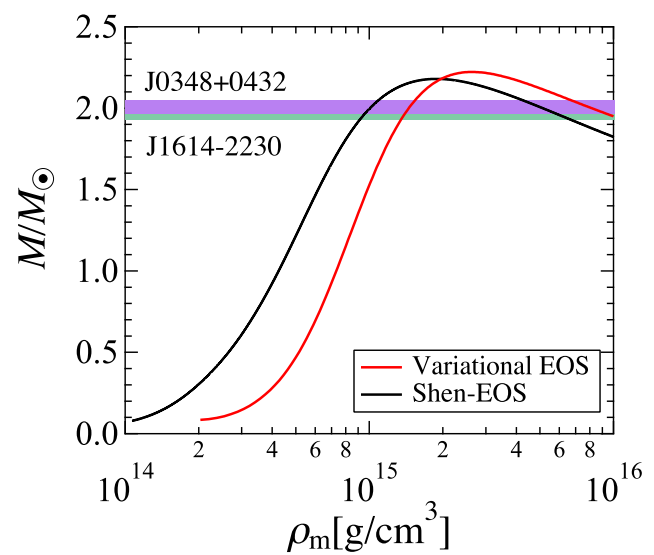

Figure 1. Gravitational masses of NSs with the present EOS and with the Shen-EOS at zero temperature as functions of the central mass density $\rho_{\mathrm{m}}$. The horizontal bands show the observational masses of PSR J16142230[9] and J0348+0432[10], respectively.

Shen-EOS. However, the obtained nuclides at various $\left(\rho, Y_{\mathrm{p}}\right)$ are rather different from those in the Shen-EOS. This difference would be due to the fact that the symmetry energy with the present EOS is smaller than that with the Shen-EOS.

\section{Application of the variational EOS of uniform nuclear matter to SNe}

In this section, we apply the present EOS of uniform nuclear matter to NSs and a SN numerical simulation. Figure 1 shows the mass of NS with the present EOS at zero temperature as a function of the central density $\rho_{\mathrm{m}}$ : Also shown is the mass with the Shen-EOS. The maximum mass with the present EOS is slightly larger than that with the Shen-EOS. Horizontal bands in Fig. 1 represent the masses of the heavy NSs J1614-2230 $\left(M=1.97 \pm 0.04 M_{\odot}\right)[9]$ and J0348+0432 $\left(M=2.01 \pm 0.04 M_{\odot}\right)$ [10]. The present EOS is consistent with those observational data. Furthermore, as reported in Ref. [4], the radii of NSs with the present EOS are consistent with the observational data analyzed in Ref. [11].

We also apply the present EOS to the simulation of core-collapse SN. Since the construction of the non-uniform EOS in our project is not finished yet, as a test application of the uniform EOS to SN simulations, we adopt the Shen-EOS for non-uniform phase, and connect it to the present uniform EOS. With use of this SN-EOS, we perform the general-relativistic spherically-symmetric (1D) simulation of the core-collapse SN. In this simulation, we neglect the weak interaction and then the electron fraction $Y_{\mathrm{e}}$ is fixed to the values at the initial condition $\left(Y_{\mathrm{e}} \gtrsim 0.4\right)$, i.e., we assume the adiabatic collapse. Details of the hydrodynamic numerical calculations are found in Ref. [12], where the corresponding simulation with the Shen-EOS is reported. Starting from the $15 M_{\odot}$ progenitor model of Ref. [13], in our adiabatic test simulation, the SN prompt explosion is successful, because the energy loss due to the neutrino emission is neglected. Figure 2 shows the density profile at bounce with the present EOS together with the one with the Shen-EOS. It is seen that the central density with the present EOS is about twice the normal density $\rho_{0}$, and is larger than that with the Shen-EOS. Correspondingly, the radius of the high-density core region with the present EOS is smaller than that with the Shen-EOS, i.e., the present EOS in this density region is softer than the Shen-EOS at $Y_{\mathrm{p}} \gtrsim 0.4$. 


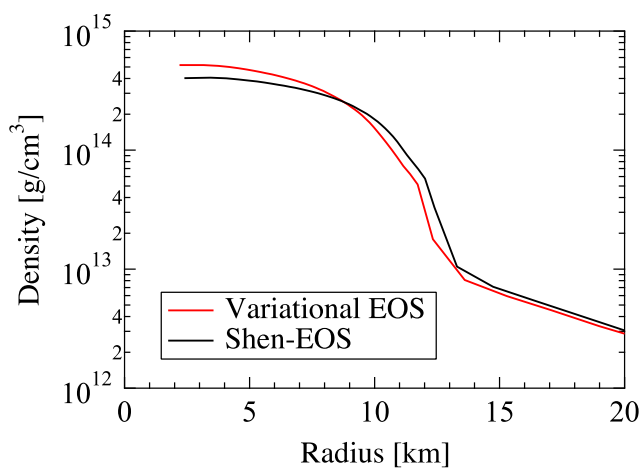

Figure 2. Density profiles of the stellar core at bounce in the 1D adiabatic core-collapse SN simulations with the present EOS (black line) and with the Shen-EOS (red line).

In fact, the explosion energy with the present EOS $\left(1.7 \times 10^{51} \mathrm{erg}\right)$ in this adiabatic simulation is larger than that with the Shen-EOS $\left(1.5 \times 10^{51} \mathrm{erg}\right)$, because the more compact stellar core releases the larger gravitational energy. This implies that the present EOS is advantageous for the SN explosions.

A more systematic study of applying this EOS to 1D core-collapse SNe, including the simulation with the neutrino transfer, will be reported elsewhere.

\section{Acknowledgements}

The numerical computations in this work were carried out on SR16000 at YITP in Kyoto University, on the GPGPU and SR16000 at the High Energy Accelerator Research Organization (KEK). This work is supported by JSPS (Nos. 21540280 and 25400275), the Grant-in-Aid for JSPS Fellows (No. 24-3275), and the Grant-in-Aid for Scientific Research on Innovative Areas of MEXT (Nos. 20105003, 20105004, 20105005).

\section{References}

[1] J. M. Lattimer and F. D. Swesty, Nucl. Phys. A 535, 331 (1991).

[2] H. Shen et al., Astrophys. J. Suppl. 197, 20 (2011).

[3] H. Kanzawa, et al., Nucl. Phys. A 791, 232 (2007).

[4] H. Togashi and M. Takano, Nucl. Phys. A 902, 53 (2013).

[5] A. Akmal, et al., Phys. Rev. C 58, 1804 (1998).

[6] K. E. Schmidt and V. R. Pandharipande, Phys. Lett. B 87, 11 (1979).

[7] A. Mukherjee, Phys. Rev. C 79, 045811 (2009).

[8] H. Kanzawa, et al., Prog. Theor. Phys. 122, 673 (2009).

[9] P. B. Demorest, et al, Nature 467, 1081 (2010),

[10] J. Antoniadis et al., Science 340, 6131 (2013).

[11] A. W. Steiner, et al., Astrophys. J. 722, 33 (2010).

[12] K. Sumiyoshi, et al., Nucl. Phys. A 730, 227 (2004).

[13] S. E. Woosley and T. Weaver, Astrophys. J. Suppl. 101, 181 (1995). 